\title{
Las cefaleas psicógenas de la infancia
}

RESUMEN: Revisión bibliográfica exhaustiva de los dolores de cabeza tensionales de la infancia. Se describe su expresión clínica, el diagnóstico diferencial, la epidemiología y los enfoques explicativos biopsicosocial y evolutivopsicodinámico.

PALABRAS CLAVE: cefalea, dolor de cabeza, psicológico, niños, epidemiología, revisión, modelos explicativos.
SUMMARY: Thorough bibliographic review about the tension type headache in childhood. It's described its clinic expression, differential diagnosis, epidemiology and the biopsychosocial and developmental-psychodinamic explanatory models.

KEY WORDS: headache, psychological, children, epidemiology, review, explanatory models

\section{Las cefáleas tensionales de la infancia}

Entendemos por cefaleas funcionales ${ }^{1}$ (jaqueca o migraña y cefalea tensional), aquellos trastornos de expresión somática en forma de dolores de cabeza, que pueden darse de manera pasajera o habitual y, en los cuales, no se detecta una causa orgánica lo suficientemente concluyente, por lo que se presupone un fuerte componente psicógeno en su etiología. Antes de diagnosticar una cefalea funcional, es preciso haber descartado previamente una etiología orgánica.

\section{Diagnóstico diferencial}

Existen dos riesgos en este proceder clínico; uno de ellos es pasar por alto un posible origen orgánico trivializando las quejas del niño; el riesgo contrario es buscar afanosamente la causa orgánica descuidando el contexto psicológico. Para diferenciar entre dolor de cabeza de tipo orgánico y funcional deben abordarse conjuntamente los aspectos médico y psicológico. Habrá de considerarse:

a. Su expresión clínica. Consiste en un análisis semiológico detallado del dolor: descripción de las crisis, localización, duración, intensidad, frecuencia, síntomas asociados, edad de aparición, etc.

b. La posibilidad de una patología orgánica. La cefalea orgánica suele ser persistente y continua, aunque a veces es intermitente. Podemos encontrarla en: 
- Trastornos asociados a la región nasal, los ojos, el cuello, los dientes u otras estructuras craneales o faciales. Por ejemplo, hay problemas oculares que, bien sea por anomalía en la convergencia o por desequilibrio entre acomodación y convergencia, cursan con dolor de cabeza.

- La sinusitis subaguda o crónica que, en ocasiones, puede pasar desapercibida. Puede justificar la cefalea.

- Tumores cerebrales. Particularmente al inicio de su desarrollo, pueden presentar episodios repentinos y agudos de dolor de cabeza, alternando con periodos de remisión. No obstante, a medida que el tumor crece cambia su intensidad y su presentación sintomática.

- La epilepsia. A veces presenta manifestaciones clínicas similares a la migraña, entre ellas, aura, ataques repentinos de duración limitada, dolor, nauseas y vómitos. Sin embargo, también hay diferencias. En la migraña los episodios son más frecuentes y duraderos y no se producen convulsiones.

- Otros procesos intracraneales como infecciones, traumatismos o hemorragias.

c. El contexto psicológico. Debemos recoger datos relevantes de la historia clínica, de la personalidad del niño, de la situación escolar y familiar, de los indicios psicopatológicos acompañantes, etc.

\section{Clasificación de los dolores de cabeza funcionales}

Los dolores de cabeza funcionales en la infancia se clasifican, generalmente, en dos categorías principales: la jaqueca o migraña infantil y la cefalea tensional. Vamos a centrarnos en la descripción clínica de ambas y en las propuestas de clasificación diagnóstica. Finalmente, se mencionarán algunas ideas de autores que critican la diferenciación entre cefalea tensional y migraña.

\subsection{La jaqueca o migraña. Expresión clínica.}

La jaqueca o migraña, del griego "hemicranea", y del latín "hemigranea", "migranea" y "migraña", es un tipo de dolor de cabeza particularmente severo, con inicio brusco y repentino, pulsátil, normalmente centrado en un solo lado de la cabeza (hemicraneal), acompañado por una combinación de síntomas neurológicos (fenómenos visuales que preceden al dolor agudo, hiperexcitabilidad sensorial que se manifiesta en fotofobia y fonofobia), gastrointestinales (náuseas, vómitos, calambres intestinales, diarreas), y autonómicos (sudoración, palpitaciones, mareo).

Los ataques, generalmente, son más cortos en el niño que en el adulto aunque pueden durar horas. Durante el ataque de migraña el niño palidece, le molesta la luz y los ruidos y evita los movimientos ya que aumentan su cefalea. Normalmente el niño se acuesta y al día siguiente se levanta sin molestias.

Algunos de los criterios que, históricamente, se han seguido para diagnosticar la jaqueca infantil son los que ahora se citan:

Para Vahlquist (4), la migraña infantil se caracteriza por cefaleas paroxísticas, separadas por intervalos libres de dolor que han de cumplir al menos dos de los 
siguientes criterios:
a. Náuseas o vómitos que acompañan al dolor de cabeza.
b. Escotoma o fenómenos visuales asociados.
c. Dolor unilateral.
d. Herencia familiar.

Prensky (5) definió la migraña infantil como dolores de cabeza recurrentes, separados por intervalos de tiempo libres de síntomas y que se acompañan de, al menos, tres de los siguientes seis síntomas:

a. Dolor abdominal, náuseas o vómitos.

b. Dolor de cabeza de localización unilateral o hemicraneal.

c. Dolor pulsátil.

d. Alivio completo después de un periodo de sueño.

e. Aura, que puede ser visual, sensorial o motor.

f. Historia familiar de migraña.

La IHS (International Headache Society) (6) divide la migraña, tal y como se ha hecho en el pasado, en dos categorías mayores: con aura (migraña clásica) y sin aura (migraña común). Propone, para pacientes menores de 15 años, los siguientes criterios que vemos en la tabla 1. La IHS-R (International Headache Society en su versión revisada) (7) propone un sistema que mantiene la especificidad pero trata de ganar en sensibilidad respecto a la población pediátrica. Los criterios revisados para el diagnóstico de jaqueca se muestran en la tabla 2.

Hay un subtipo de migraña, la migraña abdominal, que aunque está descrita desde hace más de un siglo (Gee, 1882), no está incluida en la IHS debido a que no hay suficientes huellas del dolor. El niño se ve aquejado de violentos dolores abdominales paroxísticos, difusos, asociados a náuseas y/o vómitos lo suficientemente severos como para detener su actividad normal. Van acompañados de palidez, fiebre, dolor muscular o mareo. Estos síntomas evocan un cuadro abdominal agudo, mientras que la cefalea está en segundo plano, a veces ausente y, ocasionalmente, aparece al final del dolor abdominal (8). A pesar de su peculiaridad, hay investigadores $(9,10)$ que la aceptan como una categoría más.

\subsection{La cefalea tensional. Expresión clínica.}

Cefalea de tipo tensional (tension-type headache) es el término designado por la IHS (International Headache Society) para definir lo que previamente, y según diferentes autores, ha sido llamado: cefalea de contracción muscular, cefalalgia, cefalea de estrés, cefalea esencial, cefalea ordinaria o cefalea psicógena.

Son dolores de cabeza de carácter difuso, no paroxísticos, bilaterales, no pulsátiles y que suelen describirse como "una sensación de presión”, "como una banda" o "como un casco alrededor de la cabeza". En ocasiones van acompañados de vómitos y mareo. Con frecuencia se asocian a tensiones o contracciones musculares, fundamentalmente de predominio frontal u occipital.

El dolor suele iniciarse de manera gradual y tiene unas características muy diferentes de unos casos a otros en intensidad, duración y frecuencia. Puede presentarse 
con intensidad leve, moderada o aguda. Su duración puede ir desde horas a días y su frecuencia puede ser muy variable. En su etiología los factores psicoafectivos tienen un papel preponderante, aunque no siempre es fácil la conexión entre el conflicto y la aparición de la cefalea.

La International Headache Society (IHS) distingue entre las variedades episódica (tabla 3) y crónica (tabla 4). A su vez, las divide en dos grupos: aquellas asociadas a un trastorno de los músculos pericraneales y aquellas que no están asociadas a este tipo de trastorno (7). Este criterio es contrario a la clásica asociación de la cefalea tensional con la contracción muscular.

\subsection{Críticas a la diferenciación entre cefalea de tensión y jaqueca}

La jaqueca y la cefalea tensional siguen considerándose entidades diferenciadas según la IHS (International Headache Society) aunque, en la práctica, no siempre hay una diferencia diagnóstica clara entre ambas. En algunos casos podemos encontrarnos con que la jaqueca es bilateral, sin aura, sin náuseas y sin vómitos. En otros casos se dan cefaleas tensionales que cursan con vómitos y dolor hemicraneal. También nos encontramos niños que presentan jaquecas justo después de una situación conflictiva y niños con cefaleas tensionales aparentemente desconectadas de un desencadenante conflictual.

Bakal y Kaganov (11) proponen un modelo psicobiológico basado en la cronicidad. En uno de los extremos del continuo sitúan la jaqueca y, en el otro, menos grave, la cefalea tensional, habiendo subtipos intermedios y mixtos entre uno y otro. Joffe y cols (12), en un trabajo sobre cefaleas intensas previamente diagnosticadas como tensionales o migrañosas, señalan que existe una mezcla frecuente de síntomas tradicionalmente identificados con uno de los dos tipos de cefalea. Iversen y cols (13) examinan las características clínicas de las cefaleas en una muestra de pacientes y señalan que el $62 \%$ de los pacientes con jaqueca tienen también cefalea tensional y que el $25 \%$ de los diagnosticados de tensionales tienen también episodios de jaqueca.

La IHS reconoce que los pacientes con dolores de cabeza "combinados" representan un continuo que oscila desde los puramente jaquecosos hasta los que tienen moderadas cantidades de dolor tipo tensional, aquellos que tienen la mitad de cada, los que tienen una preponderancia de cefalea tensional y los puramente tensionales. La ausencia del tipo mixto en la clasificación, la justifican diciendo que el concepto de combinado es arbitrario y, por consiguiente, es imposible establecer un grupo separado de pacientes que pudieran recibir este diagnóstico (14). Para Seshia y cols (15) no se debe rechazar la existencia de una entidad mixta. El fracaso en incorporar una entidad combinada de cefalea tensional y migrañosa fuerza a clasificar los dolores de cabeza en una u otra categoría, desvía la atención de una importante entidad clínica y, sobre todo, despista al clínico en el manejo del dolor.

Una creciente opinión clínica actual $(7,16,17,18,19,20)$ confirma el modelo de la cronicidad ya que:

a. Los síntomas de ambos tipos de cefalea (tensional y migrañosa) correlacionan en mayor medida con el grado de severidad (cronicidad) que con su especificidad sintomática. 
b. Los procesos patofisiológicos implicados en ambas pueden ser tanto vasculares como musculares y los estudios psicofisiológicos muestran grandes similitudes.

\section{Datos epidemiológicos}

Los dolores de cabeza son los síntomas somáticos de dolor más comunes en la infancia. Entre un 10 y un $25 \%$ de los niños tiene cefaleas recurrentes $(4,21,22,23,24$, $25,26,27)$. De este porcentaje, entre un 2 y un $5 \%$ son jaquecosos $(4,10,22,28,29)$. Si añadimos a estos datos el número de niños que ocasionalmente experimentan episodios de dolor de cabeza, el porcentaje total, según estudios y edades, se sitúa entre el 37 y el $76 \%(22,26,27,28,30,31,32,33)$.

A continuación presentamos algunos datos de los trabajos epidemiológicos más relevantes:

Vahlquist (4), con una población sueca de 1.236 escolares de entre 10 y 12 años, encuentra que el $4.5 \%$ sufre jaquecas, en tanto que el 13.3\% tiene cefalea tensional recurrente. Este autor, también estudia las cifras de estudiantes de escuelas secundarias (entre 16 y 19 años) y encuentra que el 7.4\% tiene ataques de jaqueca y el 19\% padece cefalea tensional recurrente.

Bille (28) hizo el estudio epidemiológico más completo hasta el momento, obteniendo datos del $95 \%$ de la población escolar entre 7 y 15 años (8993 estudiantes) de la ciudad sueca de Uppsala. Obtiene los siguientes resultados:

- El 41\% de la muestra no tenía ni había tenido nunca cefaleas, el 48\% tenía o había tenido episodios ocasionales de cefaleas de tensión, y el 11\% restante tenía o había tenido cefaleas recurrentes (entre este $11 \%, 7$ eran cefaleicos tensionales y 4 eran migrañosos).

- Entre las edades de 7 a 9 años existe una frecuencia de migraña del $2.5 \%$ en niños y $2.4 \%$ en niñas. Entre 10 y 12 años la frecuencia de la migraña es de $3.9 \%$ en niños y de $5.4 \%$ en niñas. Entre los 13 y 15 años aumenta en niños al $4 \%$ y en niñas al $6.4 \%$. A partir de la adolescencia la cefalea jaquecosa es mayor en la mujer que en el hombre. Otros tipos de cefaleas también se incrementan cada año en los dos sexos, siendo las tasas más bajas entre los preescolares.

- 73 pacientes fueron sometidos a un seguimiento (con tratamiento medicamentoso para las jaquecas) entre los 4 y los 14 años. A los 14 años, el $41 \%$ estaba asintomático, el $32 \%$ había mejorado significativamente, y el $27 \%$ estaba igual o peor.

Silampaa $(22,30)$ estudia longitudinalmente las cefaleas de niños finlandeses (evaluados a $\operatorname{los} 7$ y a los 14 años). Encontró que, de los que tenían migrañas a los 7 años, había un $37 \%$ asintomáticos, un $22 \%$ había mejorado significativamente y el $41 \%$ estaba igual o había empeorado a los 14 años. También señala que un $75 \%$ de los niños de 14 años tenían dolor de cabeza al menos una vez al mes.

Mortimer, Kay y Jaron (34), en un estudio con 1083 niños de entre 3 y 11 años, señalan que:

- No existen diferencias en cuanto a sexo en la prevalencia de la cefalea de tensión. El 56\% de los niños y el 55\% de las niñas se había quejado en algún momento 
de su vida de cefaleas de tensión (episódicas o recurrentes) siendo el 4.7\% de los niños y el $5.1 \%$ de las niñas migrañosos.

- Al valorar las cefaleas en el último año, niños y niñas se quejan con la misma frecuencia y tanto en unos como en otros aumenta con la edad. En edades entre 3 y 5 años se da en un $26.4 \%$ de los niños y un $17.5 \%$ de las niñas. Entre 5 y 7 años se da en un $36.4 \%$ de los niños y un $35 \%$ de las niñas. Entre los 7 y 9 años en un $44.5 \%$ de los niños y un $43.5 \%$ de las niñas. Entre los 9 y los 11 años en un $54.9 \%$ de los niños y un $51.6 \%$ de las niñas.

Existen pocos datos epidemiológicos sobre la incidencia familiar de migraña o cefalea tensional en los niños que se quejan de estos síntomas. Bille (28) observa que, entre los niños jaquecosos de 7 a 15 años, un $71 \%$ tienen madres jaquecosas y un 20'5 $\%$ padres jaquecosos. Oster (21) señala que el 52\% de las madres y el 39\% de los padres de niños con cefaleas (tensional o migrañosa) tienen también el síntoma. Mortimer y cols (35) indican que, en los niños con cefaleas de tipo tensional, existe una probabilidad 1'5 veces mayor que en los niños de un grupo control (sin cefaleas) de que sus madres sufran jaquecas. Este riesgo relativo alcanza en los niños con cefaleas migrañosas la probabilidad de 2.6 veces más que en el grupo control.

La relación entre otras variables sociodemográficas y cefaleas arroja resultados contrapuestos. Bille (28) no encuentra diferencias, en función de la clase social, entre niños con migraña y el grupo control. Zola (36) tampoco encuentra relación entre estos parámetros. Waters y O’Connor (37) indican que, aunque la migraña no es más frecuente entre las clases socioeconómicas altas, éstas consultan más. Golding y Butler (38) no encuentran datos positivos que relacionen la edad de la madre o el desempleo con las cefaleas infantiles, aunque si encuentran diferencias entre regiones geográficas y una estrecha asociación a la clase social baja. Zuckerman y cols (29) no distinguen entre grupos de niños con cefaleas y grupo control en las variables edad de la madre, lugar de nacimiento, nivel educativo o clase social.

Podemos concluir este apartado resumiendo que:

- Hay pocos estudios epidemiológicos y la metodología y criterios utilizados en investigación son muy variables.

- En los trabajos se ha prestado más atención a las cefaleas migrañosas que a las cefaleas tensionales.

- La mayoría de los autores coinciden en señalar que:

- Entre un 10 y un $25 \%$ de la población infantil tiene cefaleas funcionales (tensional o jaquecosa) recurrentes.

- Las cefaleas aumentan progresivamente con la edad.

- Durante la infancia, el porcentaje de niños y de niñas que se quejan de dolores de cabeza es similar.

- Los hijos de padres con cefaleas tienen más probabilidad de tener cefaleas que los hijos de padres sin cefaleas.

- Los resultados sobre la influencia de variables sociodemográficas como clase social o lugar de nacimiento son contradictorios. 


\section{Enfoques explicativos de la cefalea tensional infantil}

Marcelli y Ajuriaguerra (39), adoptando una postura etiológica conciliadora, señalan que el estudio de un trastorno se debe abordar desde distintos puntos de vista:

- Por un lado, es recomendable evaluar la patogenia potencial del contexto que rodea al niño (madre, familia, sociedad, etc.).

- Por otro lado, es recomendable situar al niño en la perspectiva diacrónica de sus etapas de maduración a fin de valorar, en el contexto de la interacción actual, el porqué del trastorno.

El primer enfoque, ambiental, permite descubrir los factores de riesgo y las características generales de una población en particular. El segundo es de naturaleza fundamentalmente individual y trata de observar la organización psicopatológica del niño.

\subsection{Enfoque biopsicosocial}

Desde esta perspectiva de trabajo, la etiología de los trastornos somatomorfos (en los que no está constatada ninguna lesión física) se considera multicausal y determinada por factores de vulnerabilidad constitucional y factores psicosociales de riesgo. Este enfoque pretende una comprensión que tenga en cuenta la interacción e influencia recíproca de los componentes biológicos, de personalidad y ambientales que se manifiestan en toda somatización.

a. Factores biológicos

Tradicionalmente se ha relacionado la cefalea tensional con la existencia de una contracción mantenida de los músculos esqueletales que se distribuyen por la cabeza y el cuello y que, al estar activos de forma casi continua, provocan el dolor. Actualmente, y como ya vimos en el apartado 2.3., se piensa que no existe necesariamente una relación entre un exceso de tensión muscular y la presencia de cefalea tensional. Tanto los factores musculares como los vasculares pueden estar implicados en la patofisiología de la cefalea tensional $(20,40)$. Joffé y cols (12) sugieren que un modelo gradual de severidad puede resultar más útil en el estudio de las cefaleas infantiles que intentar agruparlas por categorías diagnósticas.

En los trabajos de laboratorio con adultos no se verifica que los sujetos con cefaleas tensionales tengan una mayor reactividad que los grupos control en las respuestas fisiológicas relacionadas con la cefalea ante la aparición de estímulos estresantes (41).

Además del factor biológico predisponente, respecto al cual no existen conclusiones definitivas, la cefalea ha de verse como un fenómeno transaccional que afecta a la interacción sujeto-medio. Así, se intenta buscar, por un lado, características de personalidad específicas de los sujetos con cefaleas y, por otro, identificar factores psicosociales de riesgo que puedan incidir en la aparición y desarrollo del dolor de cabeza. 
b. Factores de personalidad

El intento de verificar la influencia de variables psicológicas en la cefalea se ha limitado a identificar características de personalidad aisladas, tales como neuroticismo, ansiedad, depresión, rigidez y hostilidad, que se suponía que podían diferenciar a estos niños de otros grupos control. Además, la mayoría de los datos se refieren a migrañosos y los resultados arrojan conclusiones contradictorias y poco consistentes a la hora de demostrar la anormalidad de rasgos específicos (42).

No se han realizado estudios sobre cefalea tensional y características de personalidad; tan solo algunos estudios dispersos que correlacionan cefaleas infantiles y características de timidez y perfeccionismo (43), depresión $(26,27,44)$ y ansiedad $(26,27)$ o cefaleas tensionales en adolescentes e introversión (45). Otros estudios no encuentran relación entre cefaleas y problemas de conducta $(29,38)$ y otros si encuentran esta última asociación $(26,46)$.

Carrasco (47) expone las razones por las cuales no existen en la literatura datos suficientes: en primer lugar por la falta de modelos conceptuales de los factores implicados en el desarrollo de los patrones de síntomas de dolor de cabeza durante la infancia y la adolescencia. En segundo lugar por la ausencia de estudios longitudinales que investiguen el curso de los síntomas, y en tercer lugar por la ausencia del establecimiento de diagnóstico diferencial en los criterios diagnósticos desarrollados por la Sociedad Internacional del Dolor de Cabeza (IHS).

En suma, la búsqueda científica de características de personalidad del niño cefaleico tensional es, por el momento, escasa e infructuosa.

c. Factores ambientales

El aprendizaje tiene influencia no solo en el desarrollo sino también en el mantenimiento de la somatización. Un niño que aprende que estando enfermo o quejándose de síntomas físicos consigue privilegios o evita determinadas obligaciones puede estar predispuesto a desarrollar una somatización como una estrategia de afrontamiento. Trabajos aislados han señalado la relación existente entre cefalea del niño y cefalea materna $(21,35)$.

Además de la sintomatología parental, existen factores ambientales que, a través de acontecimientos vitales negativos o de situaciones particularmente estresantes para el niño, pueden desempeñar un importante papel en el desencadenamiento del trastorno y curso del mismo. A veces es fácil identificar esos acontecimientos en casos de somatizaciones recientes de inicio agudo y el síntoma se resuelve fácilmente cuando la situación de estrés se alivia, pero otras veces el síntoma es intenso y duradero.

Los factores psicosociales de riesgo asociados a la cefalea tensional infantil no han sido estudiados con el mismo interés que en los dolores abdominales $(29,48)$ y, al igual que decíamos de las características de personalidad, la investigación es prácticamente inexistente.

A nuestro entender, el enfoque biopsicosocial ofrece un esquema explicativo lineal entre las causas externas observables y la existencia del síntoma. Esta actitud 
supone dejar al márgen las constantes interacciones entre los procesos evolutivos del niño y su ambiente.

\subsection{Enfoque evolutivo psicodinámico}

Desde un enfoque individual, la cefalea no es producto de una interacción puramente externa (en el sentido conductual estímulo-respuesta) sino que necesitan comprenderse los procesos de interiorización y relacionales en función del estadio madurativo que atraviese el niño en ese momento.

Nos centraremos en primer lugar en el contexto evolutivo en el que tiene lugar el síntoma. En segundo lugar, se revisará la diversidad de las cefaleas en función del contexto psicopatológico a través de una doble clasificación: las cefaleas neuróticas y las cefaleas de inhibición, muy diferentes en su presentación clínica y en su significado psicológico.

\subsubsection{Contexto evolutivo de la cefalea tensional}

En este trabajo nos vamos a ocupar específicamente del periodo cronológico de la escolaridad primaria, coincidente con el periodo de latencia. Describiremos esta etapa evolutiva del niño y, relacionado con las particularidades de este momento vital, abordaremos la psicodinámica de la cefalea tensional.

\subsubsection{El periodo de latencia}

S. Freud, en "El sepultamiento del complejo de Edipo" (49), señala cómo la entrada en el periodo de latencia se produce tras las decepciones sufridas por el niño, que no consigue la satisfacción edípica esperada:

"la falta de la satisfacción esperada, la continua denegación del hijo deseado, por fuerza determinará que los pequeños enamorados se extrañen de su inclinación sin esperanzas. Así el complejo de Edipo se iría al fundamento a raíz de su fracaso, como resultado de su imposibilidad interna".

Anteriormente, en "Tres ensayos para una teoría sexual" (50) sostiene que:

"en el período de latencia sexual de la infancia se edifican los poderes anímicos (asco, sentimiento de vergüenza, sentido moral) que más tarde se presentarán como inhibiciones en el camino de la pulsión sexual y angostarán su curso a la manera de diques.

La energía pulsional desviada hacia otros fines otorga importantes argumentos a las funciones culturales aunque, la sofocación pulsional, puede ser quebrada por oleadas regulares de avance del desarrollo sexual o por peculiaridades individuales (50).

Las observaciones de Freud sobre la latencia condensan los principales puntos a señalar de este periodo y permiten a otros autores posteriores apoyarse en estos elementos básicos para redefinir tanto el desarrollo psicosexual como las relaciones de objeto que se establecen en este periodo.

M. Klein (51) considera que el elemento racional tiene gran importancia en los niños de esta edad. Hay una mayor intensidad de la represión y un énfasis obsesivo sobre la realidad. Esta actitud la relaciona con las condiciones especiales de desarrollo durante este periodo: 
“en parte es resultado de su intensa preocupación por la lucha contra la masturbación, que los hace profundamente adversos a todo aquello que tenga un dejo de averiguaciones sexuales o que afecte los impulsos que están controlando con tanta dificultad" (1932, pág. 74).

Gracias a que opera una retracción, el niño normal no se ve obligado a doblegarse a las exigencias del ello. Buena parte de la carga pulsional busca soluciones de compromiso (sublimaciones, formaciones reactivas), manifestándose bajo una gran diversidad de formas indirectas (52). Los deseos libidinales dirigidos hacia los padres, en tanto que objetos de amor, son reemplazados por expresiones más sublimes de afecto: ternura, admiración, respeto (53). La energía de los nuevos investimentos sustitutorios (adquisiciones educativas, socialización exogámica, logros escolares, juegos normativos, etc.) es siempre derivada de los intereses sexuales, que siguen presentes en el inconsciente del niño y, también, en parte, en su vida consciente (52).

\subsubsection{Psicodinámica de la cefalea de la latencia}

La cefalea de la latencia es un síntoma en el encuentro del desarrollo pulsionalafectivo e intelectual del niño: por una parte, el pensamiento mágico e intuitivo debe ceder paso al pensamiento lógico, operativo, concreto y racional (54); por otra parte, las pulsiones sexuales del niño se desexualizan en favor de intereses culturales y sociales (8).

"La concienciación del razonamiento es un momento peligroso y cargado de un valor afectivo muy ambivalente. Cualquier circunstancia traumática directa o indirecta puede producir una inhibición del pensamiento racional acompañado de cefaleas, que constituyen la expresión exterior, la expresión positiva de ese fenómeno de inhibición" (55, pág. 246).

La expresión libidinal en el seno del grupo extrafamiliar permite el desarrollo de los recursos yoicos, donde las emociones sexuales del periodo edípico se calman en favor de una catectización de las metas intelectuales, sociales y escolares (56). Pero el reposo pulsional es relativo y, frecuentemente, en el inicio de la latencia podemos observar regresiones pregenitales. La actividad intelectual recientemente adquirida puede quedar asociada con el poder y rivalidad edípica o con una tentativa de seducción respecto a los padres. Las prohibiciones parentales y culturales que acompañan a los fantasmas edípicos suscitan culpas y evocan la angustia de castración. El niño, por temor a no dar satisfacción a los padres o a ser sancionado por sus deseos edípicos, pone fin a su curiosidad sexual y abandona sus investigaciones. Sensible a su fracaso y a la frustración, corre el riesgo de evolucionar hacia una inhibición intelectual acompañada de cefaleas, que le permitirá la negación de realidades intolerables para él (57). Los beneficios primarios conseguidos por la cefalea son, en primer lugar, la evitación del conflicto surgido de la ambivalencia y, en segundo lugar, la libidinización masoquista del conflicto (58).

Las cefaleas episódicas de la latencia, dentro de un contexto edípico, normalmente indican la utilización de un sistema regresivo tras el cual subyacen fantasías representables y verbalizables. 
Por el contrario, cuando las cefaleas son precoces y crónicas, el aparato mental no ha sido apenas utilizado en el período de latencia y las actividades fantasmáticas y oníricas han sido casi nulas en razón del obstáculo puesto a la evolución mental. En el origen de estos estados se halla una ausencia o extrema pobreza del investimento libidinal que imposibilita al niño para el desarrollo de una actividad autónoma sublimada como es la intelectual. Las cefaleas recurrentes en el contexto de una disarmonía evolutiva ${ }^{2}$ van asociadas a graves problemas de la organización psíquica del niño que perturban su desarrollo, sus posibilidades de adquisición y sus relaciones objetales.

En definitiva, el significado evolutivo de la cefalea es variado y ha de estudiarse además el contexto psicológico en el que se inscribe.

\subsubsection{Contexto psicopatológico de la cefalea tensional}

Acabamos de ver que las cefaleas tensionales de la latencia no son unívocas y no existe una explicación psicodinámica global para todas ellas. Su sentido depende del contexto psicológico y del funcionamiento mental predominante en el niño.

El texto de Freud Inhibición, Síntoma y Angustia (62) ha supuesto para nosotros una valiosa aportación a la hora de entender la expresión psicopatológica de las cefaleas en la latencia. Dice que:

"muchas inhibiciones son una renuncia a una función porque a raíz de su ejercicio se desarrollaría un síntoma de angustia" (pág. 84), "limitan una función yoica, ya sea por precaución o a consecuencia de un empobrecimiento de energía” (pág. 86).

Distingue varios tipos de inhibiciones:

a. Inhibiciones específicas (neuróticas). La función yoica de un órgano se deteriora cuando aumenta su erogeneidad, su significación sexual. Aquí el yo renuncia a estas funciones para evitar un conflicto con el ello.

b. Inhibiciones al servicio de la autopunición. El yo tiene prohibido realizar determinadas actividades (ej. intelectuales) porque le proporcionarían provecho y éxito, algo que el severo superyo le ha denegado. El yo renuncia a esas operaciones a fin de no entrar en conflicto con el superyo.

c. Inhibiciones generales del yo. El yo se encuentra absorto por una labor psíquica de particular gravedad y se empobrece tanto la energía de que puede disponer, que se ve obligado a restringir su gasto en muchos lugares.

En este pequeño recorrido, Freud nos desvela diferentes contextos psicopatológicos en los que ubicar la cefalea que, en sí misma, representa una inhibición de la función del pensar. Puede ser un trastorno banal secundariamente investido y utilizado, un síntoma conversivo, una señal de alarma en estados depresivos graves, etc. Se presenta dentro de las estructuras mentales más diversas.

Sirol (8), basándose en Kreisler (63), distingue entre el contexto neurótico y el contexto de inhibición. Vamos a apoyarnos en estas dos referencias.

\subsubsection{Cefaleas neuróticas}

Estas cefaleas, habituales en niños emotivos y ansiosos, cubren una amplia gama que va desde las cefaleas imitadas del niño fóbico que busca, de manera episódica, algún 
beneficio secundario hasta aquellas que se instalan insidiosamente y van acompañadas de otras emergencias neuróticas.

Las cefaleas de beneficio secundario tienen el carácter de explotación de las ventajas del dolor. A través de mecanismos regresivos el niño consigue, por ejemplo, un exceso de atención del entorno o cuidados privilegiados. En muchos casos, la familia está más pendiente del niño, le mima más, le vigila y es objeto de repetidas exploraciones. Otras veces, la cefalea aparece asociada a una circunstancia determinada (ir al colegio, hacer trabajos desagradables, etc.). En este caso hay una utilización consciente del síntoma para evitar hacer algo que no le gusta.

A veces no se sabe muy bien donde está el límite y puede confundirse la cefalea de tensión con una simulación de dolor. El entorno sospecha de esa inautenticidad y escuchamos de los padres frases como: "yo ya no se si lo hace para llamar la atención", "no se si le duele de verdad", "si no le hacemos caso enseguida se le pasa", "le das cualquier cosa y se le pasa", etc.

La predisposición del niño a los síntomas físicos puede ser reforzada por las actitudes parentales o por la presencia de modelos de identificación familiar (principalmente la madre) que también se quejan de dolores de cabeza. La cefalea se instala progresivamente debido a que los padres conceden un interés desmedido a la cabeza del hijo, que se convierte en un lugar privilegiado.

Cuando el dolor de cabeza llega a ser algo reiterado, investido y cargado de significados, con el cual se obtiene una serie de beneficios o se evita una serie de actividades displacenteras, generalmente, podemos observar otros signos neuróticos (manifestaciones de ansiedad, miedos, obsesiones, características histeriformes) entrelazados e interdependientes con el dolor de cabeza.

Los síntomas neuróticos deben investigarse. Podemos encontrar elementos obsesivos enquistados, persistencia de fobias que evolutivamente ya no son normales o problemas con el sueño (pesadillas, dificultades para dormir solo). En estos casos, es conveniente replantearse el diagnóstico estructural de la organización mental prevalente.

\subsubsection{Cefaleas de inhibición}

En este contexto, el niño sufre un empobrecimiento intelectual, afectivo y pulsional, acompañado del bloqueo doloroso del pensamiento. La cefalea, consecuencia de la inhibición, constituye un grave síntoma que pone de manifiesto la confluencia entre la anormal evolución psicoafectiva y libidinal del niño y los mecanismos psicopatológicos que corresponden a su medio entorno.

Estos dolores de cabeza aparecen ante un exceso de exigencia por parte del medio. Por un lado están las tendencias internas del sujeto y, por otro, las demandas del exterior. Las condiciones exteriores que facilitan su aparición pueden ser diversas:

- Situaciones de estrés o sobreexcitación que originan un exceso de tensión psíquica. Por ejemplo, asociadas a exámenes escolares o exceso de actividades en el programa diario del niño. Otras circunstancias pueden ser la exigüidad de la vivienda, los ruidos, la agitación familiar, etc. Estas cefaleas frecuentemente desaparecen en periodos 
de vacaciones. A veces, simplemente la reorganización del tiempo o la planificación más ordenada de la vida cotidiana es suficiente para que la inhibición desaparezca y la cefalea remita.

- Ambiente familiar muy exigente. Se educa al niño en un clima de excesiva demanda hacia él. Ha de ser "perfecto", "el mejor", y no se permiten los fallos. Para los padres es preciso hacer todo cuanto antes y eso aumenta las dificultades del niño. Esta actitud parental se refleja en un desarrollo hipermaduro del niño que, desde el principio, evoluciona precozmente en todas las áreas (locomoción, lenguaje, esfinteres, alimentación, escolaridad, etc.). Bajo estas características se camufla un superyo sádico que no tolera un desliz y parece siempre insatisfecho. Las manifestaciones de agresividad o de rebeldía naturales del niño son difícilmente toleradas por su ambiente y se anula su espontaneidad.

- Miedo a sobrepasar a los padres y a perder su amor. El triunfo es percibido como una situación de peligro porque el niño no se atreve a enfrentarse y rivalizar con el padre y superarlo. Sufre entonces de donde cree que hace mal, la cabeza. El yo inhibe su actividad para no entrar en conflicto con el superyo severo. Son lo que Freud llamó inhibiciones al servicio de la autopunición.

Las condiciones enumeradas son directa o indirectamente responsables de la inhibición, pero en cualquier caso demuestran la dependencia del niño y la fragilidad de su investimento intelectual (57). En algunos casos la comprensión de la situación que reactiva la cefalea permite un abordaje terapéutico eficaz. En los casos donde el conflicto está gravemente internalizado conviene precisar la organización patológica en coincidencia con la cefalea de inhibición. Pensamos que este tipo de inhibición corresponde a lo que Freud denominó inhibiciones generales del yo o, en la terminología de P. Marty, estados operatorios acompañados de una depresión no mentalizada.

Bourdier (64) considera que el "síndrome: trastornos fobo-obsesivos-trastornos del sueño-cefalea", sería ya un estado premórbido de la infancia situado en un cruce de caminos entre la neurosis, la psicosis, la organización caracterial y la enfermedad orgánica. Según Marty (55), en esta cefalea no existe ninguna organización neurótica pero tampoco psicótica. La diferencia respecto a lo normal estaría representada, sobre todo, por una ausencia de libertad fantasmática y de la vida onírica, un empobrecimiento de los intercambios interpersonales y una esclerosis de la expresión verbal (pensamiento operatorio). En los casos más graves plantea el problema del déficit de preconsciente en un periodo precoz del desarrollo.

\footnotetext{
NOTAS.-

${ }^{1}$ El término “funcional” lo acuñó Von Bergman (1). La patología funcional, a primera vista, parecería limitarse tan solo al mero funcionamiento, sin implicar en modo alguno la integridad estructural del órgano. En ese sentido, lo funcional se opondría a lo lesional orgánico. Una consideración más detenida obligaría a relativizar la distinción, admitiendo las hipótesis que sugieren que, a veces, lo funcional es el paso previo a una lesión. Además, cuando decimos que algo es funcional decimos también, en realidad, que es disfuncional, y ese es el sentido que querría transmitir con el término; que una función se haya alterada sin que una lesión orgánica lo justifique. Tal y como señalan López Sanchez (2) o Ridruejo (3), lo funcional constituye un tránsito entre lo psíquico y lo somático, y en ese tránsito, precisamente, pretende inscribirse la psicosomática.
} 


\begin{abstract}
${ }^{2}$ El concepto de disarmonía evolutiva, utilizado por autores como Lebovici (59) o Ajuriaguerra (60), pretende señalar el desajuste que se establece entre las lineas en curso de maduración. Este concepto deriva de lo que Anna Freud (61) denomina lineas de desarrollo; ella señala que estas lineas "representan el resultado de la interacción entre el desarrollo del yo, del superyo y de sus reacciones frente a las influencias del medio, es decir, entre los procesos de maduración, adaptación y estructuración" (pág. 56), "trazan el gradual crecimiento del niño desde las actitudes dependientes, irracionales, determinadas por el ello y los objetos hacia un mayor control yoico del mundo interno y externo" (pág. 55). El efecto desorganizador de la disarmonía surge cuando el yo y el superyo no poseen la madurez suficiente para controlar las pulsiones parciales pregenitales y agresivas.
\end{abstract}

1. Migraña sin aura

A. Al menos 5 ataques que cumplan B-D.

B. El ataque de cefalea dura entre 2 y 48 horas.

C. La cefalea cumple al menos dos de los siguientes:

1. Localización unilateral.

2. De calidad pulsátil.

3. Intensidad de moderada a severa (inhibe o impide la actividad diaria).

4. Se agrava subiendo escaleras o con una actividad física rutinaria similar.

D. Durante el episodio se da al menos una de las siguientes: 1. Nausea o vómitos.

2. Fotofobia o fonofobia.

2. Migraña con aura (descrita como un trastorno recurrente idiopático en el que el dolor dura entre 2 y 48 horas)

A. Al menos 2 ataques que cumplan B.

B. Al menos 3 de las siguientes:

1. Uno o más síntomas de aura reversibles en su totalidad, indicando una disfunción cortical focal y/o disfunción del tronco cerebral.

2. Al menos un aura desarrollado gradualmente durante más de 4 minutos o dos o más síntomas que ocurran sucesivamente.

3. Que ningún aura dure más de 60 minutos.

4. El dolor sigue al aura con un intervalo libre menor de 60 minutos (puede incluso comenzar antes o simultanemente al aura).

Tabla 1. Criterios diagnósticos de la migraña pediátrica IHS, 1988 (adaptado de Winner y cols, 1995, pág. 408) 
1. Migraña sin aura

A. Al menos cinco ataques que cumplan B-D.

B. El ataque de cefalea dura entre 30 minutos y 48 horas.

C. La cefalea tiene al menos dos de los siguientes:

1. Localización bilateral (frontal/temporal) o unilateral.

2. Calidad pulsátil.

3. Intensidad de moderada a severa.

4. Se agrava con la actividad física rutinaria.

D. Durante el episodio se da al menos una de las siguientes:

1. Nauseas y/o vómitos.

2. Fotofobia y/o fonofobia.

2. Migraña con aura (descrita como trastorno recurrente idiopático en el que el dolor de cabeza dura entre 30 minutos y 48 horas).

A. Al menos dos ataques que cumplan B.

B. Al menos tres de las siguientes:

1. Uno o más síntomas de aura reversibles en su totalidad indicando una disfunción cortical focal y/o disfunción del tronco cerebral.

2. Al menos un aura desarrollado gradualmente durante más de 4 minutos, o dos o más síntomas que ocurran sucesivamente. 3. Que ningún aura dure más de 60 minutos.

4. La cefalea sigue al aura con un intervalo libre de menos de 60 minutos.

Tabla 2. Criterios diagnósticos de la migraña pediátrica (IHS-R, 1995) (adaptado de Winner y cols, 1995, pág. 408) 
Cefalea episódica de tipo tensional

A. Al menos diez episodios previos que cumplan los criterios B-D, con una frecuencia de dolor promedio menor de 15 días al mes $(180$ días al año).

B. Que el dolor dure entre 30 minutos y 7 días.

C. Al menos dos de las siguientes características de dolor:

1. Calidad de "presión" o "tirantez"; no pulsátil.

2. Intensidad de leve a moderada (puede inhibir, aunque no impedir actividades).

3. Localización bilateral.

4. No se agrava subiendo escaleras o realizando actividades físicas rutinarias similares.

D. Que se den los dos siguientes criterios:

1. Sin nauseas o vómitos (aunque en la anorexia pueden darse). 2. La fotofobia o fonofobia están ausentes o bien uno de los dos está presente y el otro ausente.

Cefalea tensional episódica asociada a un trastorno de los músculos pericraneales. A. Se cumplen los criterios de cefalea episódica de tipo tensional. B. Al menos uno de los siguientes criterios:

1. Incremento de la sensibilidad de los músculos pericraneales.

2. Incremento del nivel electromiográfico de los músculos pericraneales.

Cefalea tensional episódica no asociada a un trastorno de los músculos pericraneales. A. Se cumplen los criterios de cefalea episódica de tipo tensional.

B. No se incrementa la sensibilidad ni la actividad electromiográfica de los músculos pericraneales.

Tabla 3. Criterios diagnósticos de la cefalea tensional episódica (IHS, 1988) (adaptado de Silberstein, 1993, pág. 1645) 
Cefalea tensional crónica

A. Frecuencia de dolor promedio de más de 15 días por mes durante más de 6 meses, cumpliendo los criterios B-D.

B. Al menos 2 de las siguientes características:

1. Calidad del dolor presionante o tirante.

2. Intensidad de leve a moderada (puede inhibir pero no impedir las actividades).

3. Localización bilateral.

4. No se agrava por subir escaleras o realizar similares actividades físicas rutinarias.

C. Que se den los dos siguientes criterios:

1. Sin vómitos.

2. No más de una de las siguientes: nauseas, fotofobia o fonofobia.

Cefalea crónica de tensión asociada a un trastorno de los músculos pericraneales A. Que cumpla los criterios de cefalea tensional crónica.

B. Al menos uno de los siguientes:

1. Incremento de la sensibilidad de los músculos pericraneales. 2. Incremento del nivel EMG de los músculos pericraneales.

Cefalea crónica de tensión no asociada a un trastorno de los músculos pericraneales.

A. Que cumpla los criterios de cefalea tensional crónica.

B. No hay un incremento de la sensibilidad o de la actividad electromiográfica en los músculos pericraneales.

Tabla 4. Criterios diagnósticos de la cefalea tensional crónica (IHS, 1988) (adaptado de Silberstein, 1993, pág. 1645) 


\section{BIBLIOGRAFIA}

(1) Von Bergman, G., "Concepto e importancia de la biología, patología y terapéutica funcionales", Folia Clínica Internacional, 1970, 20, 3.

(2) López Sánchez, J.M., "Del síntoma hipocondríaco al síntoma psicosomático”. En J.M. López Sánchez (Ed), Resúmenes de Patología Psicosomática, Vol. 2, pp. 331-343. Granada, Círculo de Estudios Psicopatológicos, 1989.

(3) Ridruejo, P., Psicología médica, Barcelona, McGraw-Hill-Interamericana, 1996.

(4) Vahlquist, B., "Migraine in children", International Archives of Allergy, 1955, 7, pp. 348-355.

(5) Prensky, A.L., "Migraine and migrainous variants in pediatric patients", Pediatric Clinics of North America, 1976, 23 (3), pp. 461-471.

(6) Winner, P., y otros, "Classification of pediatric migraine: proposed revisions to the IHS criteria", Headache, 1995, 35 (7), pp. 407-410.

(7) Silberstein, S.D., "Tension-type and chronic daily headache", Neurology, 1993, 43, pp. 1644-1649.

(8) Sirol, F., "Las cefaleas recurrentes de la última infancia y de la adolescencia". En S. Lebovici, R. Diatkine y M. Soule (Eds), Tratado de Psiquiatría del Niño y del Adolescente, Vol. 4, Madrid, Biblioteca Nueva, 1990.

(9) Symon, D.N.K.; Russell, G., "Abnormal migraine: a childhood syndrome defined", Cephalalgia, 1986, 6, pp. 223-228.

(10) Mortimer, M.J.; Kay, J.; Jaron, A., "Clinical epidemiology of childhood abdominal migraine in an urban general practice", Developmental Medicine and Child Neurology, 1993, 35, pp. 243-248.

(11) Bakak, D.A.; Kaganov, J.A., "Symptom characteristics for chronic and non chronic headache sufferers", Headache, 1979, 19, pp. 285-289.

(12) Joffe, R.; Bakal, D.A.; Kaganov, J.A., "A self observation study of headache symptoms in children", Headache, 1983, 23, pp. 20-25.

(13) Iversen, H.K., y otros, "Clinical characteristics of migraine and episodic tension-type headache in relation to old and new diagnostic criteria", Headache, 1990, 30, pp. 514-519.

(14) Saper, J.R., "Daily chronic headache", Neurologic Clinics, 1990, 8 (4), pp. 891-901.

(15) Seshia, S.S., y otros, "International headache society criteria and childhood headache", Developmental Medicine and Child Neurology, 1994, 36, pp. 419-428.

(16) Nattero, G., y otros, "Idiophatic headaches: relationship to life events", Headache, 1986, 26, pp. 503-508.

(17) Appelbaum, K.A., y otros, "The pain behavior questionnaire (PBQ): a global report of pain behavior in chronic headache", Headache, 1988, 28, pp. 53-58.

(18) Martínez Sánchez, F., y otros, "Análisis psicométrico de un cuestionario diagnóstico de cefaleas funcionales. Una contribución a los modelos explicativos basados en la cronicidad", Análisis y Modificación de Conducta, 1992, 18 (59), pp. 397-412.

(19) Gladstein, J., y otros, "Diagnoses and symptom patterns in children presenting to a pediatric headache clinic", Headache, 1993, 33, pp. 497-500.

(20) Silberstein, S.D., "Tension type headaches", Headache, 1994, 34 (8), S2-S7.

(21) Oster, J., "Recurrent abdominal pain, headache and limb pains in children and adolescents", Pediatrics, 1972, 50 (3), pp. 429-436.

(22) Silampaa, M., "Changes in prevalence of migraine and other headaches during the first seven years of school", Headache, 1983, 23, pp. 15-19. 
(23) Hodges, K., y otros, "Life events occurring in families of children with recurrent abdominal pain”, Journal of Psychosomatic Research, 1984, 28 (3), pp. 185-188.

(24) Aro, H., "Life stress and psychosomatic symptoms among 14 to 16 year old finnish adolescents", Psychological Medicine, 1987, 17, pp. 191-201.

(25) Garber, J.; Walker, L.S.; Zeman J., "Somatization symptoms in a community sample of children and adolescents: further validation of the Children's Somatization Inventory", Journal of Consulting and Clinical Psychology, 1991, 3 (4), pp. 588-595.

(26) Egger, H.L.; Angold, A.; Costello, E.J., "Headaches and psychopathology in children and adolescents", Journal of the American Academy of Child and Adolescent Psychiatry, 1998, 37 (9), pp. 951-958.

(27) Egger, H.L., y otros, "Somatic complaints and psychopathology in children and adolescents: stomach aches, musculoskeletal pains and headaches", Journal of the American Academy of Child and Adolescent Psychiatry, 1999, 38 (7), pp. 852-860.

(28) Bill, B., "Migraine in school children", Acta Paediatrica, 1962, 51, suppl. 136.

(29) Zuckerman, B.; Stevenson, J.; Biley, V., "Stomachaches and headaches in a community sample of preschool children”, Pediatrics, 1987, 79 (5), pp. 677-682.

(30) Silampaa, M., "Prevalence of migraine and other headaches in finnish children starting school", Headache, 1976, 15, pp. 288-290.

(31) Hockaday, J.M., "Headache in children”, British Journal of Hospital Medicine, 1982, 27, pp. 383-391.

(32) Collin, C.; Hockaday, J.M.; Waters, W.E., "Headache and school absence", Archives of Disease in Childhood, 1985, 60, pp. 245-247.

(33) Barea, L.M.; Tannhauser, M.; Rotta, N.T., "An epidemiologic study of headache among children and adolescents of southern Brasil", Cephalalgia, 1996, 16, pp. 545-549.

(34) Mortimer, M.J.; Kay, J.; Jaron, A., "Epidemiology of headache and childhood migraine in an urban general practice using Ad Hoc, Vahlquist, and IHS criteria", Developmental Medicine and Child Neurology, 1992, 34, pp. 1095-1101.

(35) Mortimer, M.J., y otros, "Does a history of maternal migraine or depression predispose children to headache and stomachache?", Headache, 1992, 32, pp. 353-355.

(36) Zola, I.K., "Culture and symptoms. An analysis of patients presenting complaints", American Society Review, 1966, 31, pp. 615.

(37) Waters, W.E.; O’Connor, P.J., "Epidemiology of headache and migraine in women", Journal of Neurol. Neurosur. Psychiatr., 1971, 34, pp. 148-153

(38) Golding, J.; Butler, N.R., Headaches and stomachaches. En J. Golding y N.R. Butler (Eds), From birth to Five (cap. 9). Oxford, Pergamon Press, 1986.

(39) Marcelli, D.; Ajuriaguerra, J., Psicopatología del niño, Barcelona, Masson. $3^{\text {a }}$ ed., 1996.

(40) Labbe, E.E., "Childhood muscle contraction headache: current issues in assessment and treatment", Headache, 1988, 28, pp. 430-434.

(41) De la Puente, M.L., Efectos diferenciales del entrenamiento en biofeedback EMG frontal y de la inoculación del estrés en el tratamiento de las cefaleas funcionales, Madrid, Universidad Complutense, 1990.

(42) Merikangas, K.R., "Psychopatology and headache syndromes in the community", Headache, 1994, 34 (8), S17-S22.

(43) Kowal, A.; Pritchard, D., "Psychological characteristics of children who suffer from headache: a research note", Journal of Child Psychology and Psychiatry, 1990, 31, pp. 637-649. 
(44) Pine, D.S.; Cohen, P.; Brooke, J., "The association between major depression and headache: results of a longitudinal epidemiologic study in youth", Journal of Child and Adolescent Psychopharmacology, 1996, 6, pp. 153-164.

(45) Rangaswamy, K., "Tension headache in adolescents", Journal of Psychological Research, 1982, 26, pp. 70-72.

(46) Taylor, D.C.; y otros, "Somatization and the vocabulary of everyday bodily experiences and concerns: a community study of adolescents", Journal of the American Academy of Child and Adolescent Psychiatry, 1996, 35, pp. 491-499.

(47) Carrasco, M.A., "Dolor de cabeza en niños: una revisión”, Psiquis, 2000, 21 (3), pp. 50-63.

(48) Garralda, M.E., "A selective review of child psychiatric syndromes with a somatic presentation", British Journal of Psychiatry, 1992, 161, pp. 759-773.

(49) Freud, S., El sepultamiento del complejo de edipo, Buenos Aires, Amorrortu, 1924, 19, p. 181.

(50) Freud, S., Tres ensayos de teoría sexual, Buenos Aires, Amorrortu, 1905.

(51) Klein, M., La técnica del análisis en el periodo de latencia, Barcelona, Paidós, 1932, 2, pp. 74-94.

(52) Widlocher, D., Estudios sobre sexualidad humana. Madrid, Editorial Morata, 1967.

(53) Bergeret, J., Manual de psicología patológica, Barcelona, Masson. 2a ed., 1990).

(54) Piaget, J., Seis estudios de psicología, Barcelona, Ariel, 1983.

(55) Marty, P., "Aspects psychodynamiques de l'étude clinique de quelques cas de céphalalgies", Revue Française de Psychanalyse, 1951, 15 (2), pp. 216-252.

(56) Kreisler, L., El Niño psicosomático, Buenos Aires, Huemul, 1975.

(57) Danon-Boileau, H.; Lab, P., "L'Inhibition intellectuelle", La Psychiatrie de l'enfant, 1962, 5, pp. 43-173.

(58) Cobo, C., Paidopsiquiatría dinámica, Madrid, Servicio Científico "Roche", 1983.

(59) Lebovici, S., "La histeria en el niño y en el adolescente". En S. Lebovici, R. Diatkine y M. Soule (Eds), Tratado de Psiquiatría del Niño y del Adolescente, Vol. 3, Madrid, Biblioteca Nueva, 1989.

(60) Ajuriaguerra, J., Manual de psiquiatría infantil, Barcelona, Masson, $4^{\mathrm{a}}$ ed., 1993.

(61) Freud, A., Normalidad y patología en la niñez, Buenos Aires, Paidos, $5^{\text {a }}$ reimpresión, 1984.

(62) Freud, S., Inhibición, síntoma y angustia, Buenos Aires, Amorrortu, 1926, 20, pp. 71-164.

(63) Kreisler, L., "L'abord psychologique de la céphalée essentielle de l'enfant et de l'adolescent", Gazzette Médicale de France, 1968, 75 (3), pp. 507-520.

(64) Bourdier, P., "La céphalée de l'enfant ou contribution à l'étude des états prémorbides de l'enfance", Revue Française de Psychanalyse, 1962, 26 (6), pp. 633-654.

*Psicólogo Clínico. Doctor en Psicología

Lugar de trabajo: Hospital Universitario Virgen del Rocío. Sevilla.

Correspondencia: juanotero01@mixmail.com

Fecha de recepción: 8-01-02 